Supporting Information for

\title{
Multifunctional Graphene-Oxide-Reinforced Dissolvable Polymeric \\ Microneedles for Transdermal Drug Delivery
}

Yongli Chen,, ac,d Yikun Yang, ${ }^{\mathrm{b}}$ Yiwen Xian, ${ }^{\mathrm{d}}$ Parbeen Singh, ${ }^{\mathrm{a}, \mathrm{c}, \mathrm{d}}$ Jinglong Feng, ${ }^{\mathrm{a}, \mathrm{d}}$

Shufen Cui, ${ }^{\mathrm{d}}$ Andrew Carrier, ${ }^{\mathrm{e}}$ Ken Oakes, ${ }^{\mathrm{f}}$ Tiangang Luan, ${ }^{\mathrm{c}}$ and Xu Zhange,

aPostdoctoral Innovation Practice Base, Shenzhen Polytechnic, Shenzhen, 518055,

China.

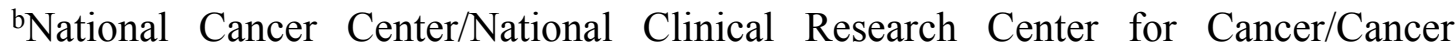

Hospital \& Shenzhen Hospital, Chinese Academy of Medical Sciences and Peking Union Medical College, Shenzhen, 518116, China

${ }^{\mathrm{c} M O E}$ Key Laboratory of Aquatic Product Safety, School of Marine Sciences, Sun Yat-sen University, Guangzhou 510275, China

${ }^{\mathrm{d}}$ Department of Biological Applied Engineering, Shenzhen Key Laboratory of Fermentation Purification and Analysis, Shenzhen Polytechnic, Shenzhen, 518055, China

${ }^{\mathrm{e}}$ Department of Chemistry, fDepartment of Biology, Cape Breton University 1250

Grand Lake Road, Sydney, Nova Scotia, B1P 6L2, Canada

${ }^{*}$ Corresponding author: xu zhang(cbu.ca (X. Z.) 


\section{Experimental}

\section{In vitro dissolution of FITC from PVP and GO-PVP MNs}

Fluorescein isothiocyanate (FITC, $20 \mathrm{mM}$ ) was loaded into PVP and GO-PVP MNs to compare their dissolution kinetics. The MNs were incubated in $2 \mathrm{~mL}$ of deionized water at $37{ }^{\circ} \mathrm{C}$, and $100 \mu \mathrm{L}$ of the solution was removed and replaced with fresh water at predetermined intervals. The removed aliquots were placed in a 96 well plate and measured using a microplate reader (SpectraMax M5e, Molecular Devices, San Jose, CA, USA) with excitation and emission at 494 and $520 \mathrm{~nm}$, respectively, and calibrated against a standard curve for FITC. The measurements were performed in triplicate.

\section{Cell culture}

U937 cells were cultured in RPMI-1640 medium supplemented with 10\% FBS, 100 $\mu \mathrm{g} / \mathrm{mL}$ penicillin, and $100 \mu \mathrm{g} / \mathrm{mL}$ streptomycin. CCC-ESF cells and B16F10 cells were cultured in DMEM medium supplemented with $10 \% \mathrm{FBS}, 100 \mu \mathrm{g} / \mathrm{mL}$ penicillin, and $100 \mu \mathrm{g} / \mathrm{mL}$ streptomycin. The cells were cultured at $37{ }^{\circ} \mathrm{C}$ in $5 \% \mathrm{CO}_{2}$ and passaged every $2 \mathrm{~d}$.

\section{ELISA assay}

The human monocyte cell line U937 was differentiated into macrophages using PMA (phorbol-12-myristate 13-acetate) and LPS (lipopolysaccharides). In detail, U937 cells were seeded in a 24 well plate at a density of $5 \times 10^{5}$ cells/well. The macrophage-like state (M0) was obtained by treating U937 monocytes for $48 \mathrm{~h}$ with $100 \mathrm{ng} / \mathrm{ml}$ PMA in each well. Then, the medium was changed with fresh medium 
containing $1 \mu \mathrm{g} / \mathrm{ml}$ LPS and incubated for $24 \mathrm{~h}$ to activate M0 to form M1 macrophages, simulating an inflammatory or infectious environment. Afterwards, M1 cells were treated with PVP MN solutions with different GO concentrations for $6 \mathrm{~h}$. The incubation culture media were collected and centrifuged to remove the cell debris and GO. The pro inflammatory cytokines in the supernatant were analyzed, including tumor necrosis factor-alpha (TNF $\alpha$ ) and two interleukins (IL-1 $\beta$ and IL-6), using ELISA kits following the manufacturer's instructions.

\section{Cytotoxicity assay}

The cell viability was determined by MTT assay. CCC-ESF cells were seeded on 96 well plates at a density of $3 \times 10^{4}$ cells/well. After $10 \mathrm{~h}$ of cell growth the medium was changed with fresh medium containing $\mathrm{MN}$ or GO-MN solutions. After $24 \mathrm{~h}$ treatment, $100 \mu \mathrm{L}$ MTT solution $(5 \mathrm{mg} / \mathrm{mL})$ was added into each well and incubated for $4 \mathrm{~h}$. The solution was then replaced by $100 \mu \mathrm{L}$ DMSO. The 96 well plate was gently shaken for $5 \mathrm{~s}$, and measured under the microplate reader (SpectraMax M5e, Molecular Devices, San Jose, CA, USA). The measurement was performed in triplicate.

\section{Toxicity evaluation}

The histological and hepatotoxicity study were performed to evaluate the toxicity of the GO-microneedles toward mice. For histological evaluation, on the day after the last treatment, two mice from each group were sacrificed, and sections of each of the main organs were collected and stained with hematoxylin and eosin for toxicity evaluation. For hepatotoxicity study, on the day after the last treatment, the blood 
from each group of mice was collected directly from the eyes. The blood samples were stored at $4{ }^{\circ} \mathrm{C}$ overnight and centrifuged at $3000 \mathrm{rpm}$ for $20 \mathrm{~min}$ to separate the plasma. The plasma levels of AST and ALT in mice were assayed according to the protocols provided by the manufacturer. The absorbance was measured with a plate reader (SpectraMax M5e, Molecular Devices, San Jose, CA, USA) at $510 \mathrm{~nm}$. 


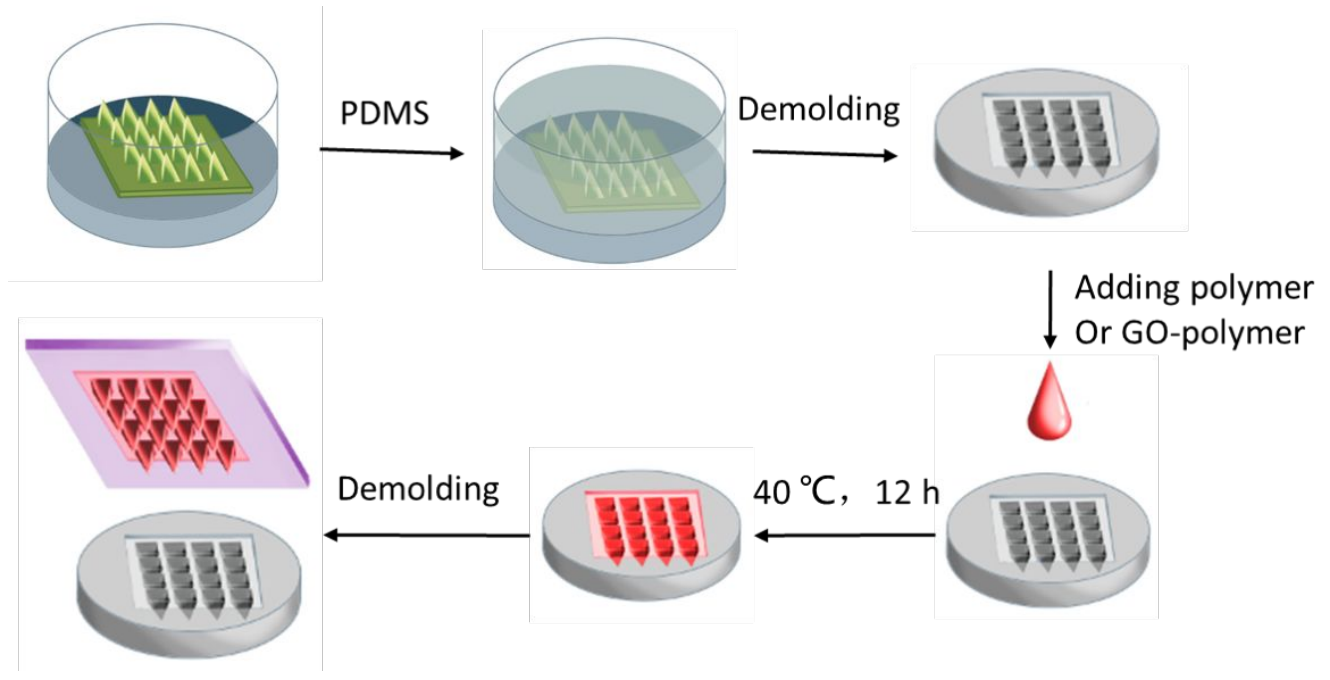

Scheme S1. The microneedle patch fabrication process.
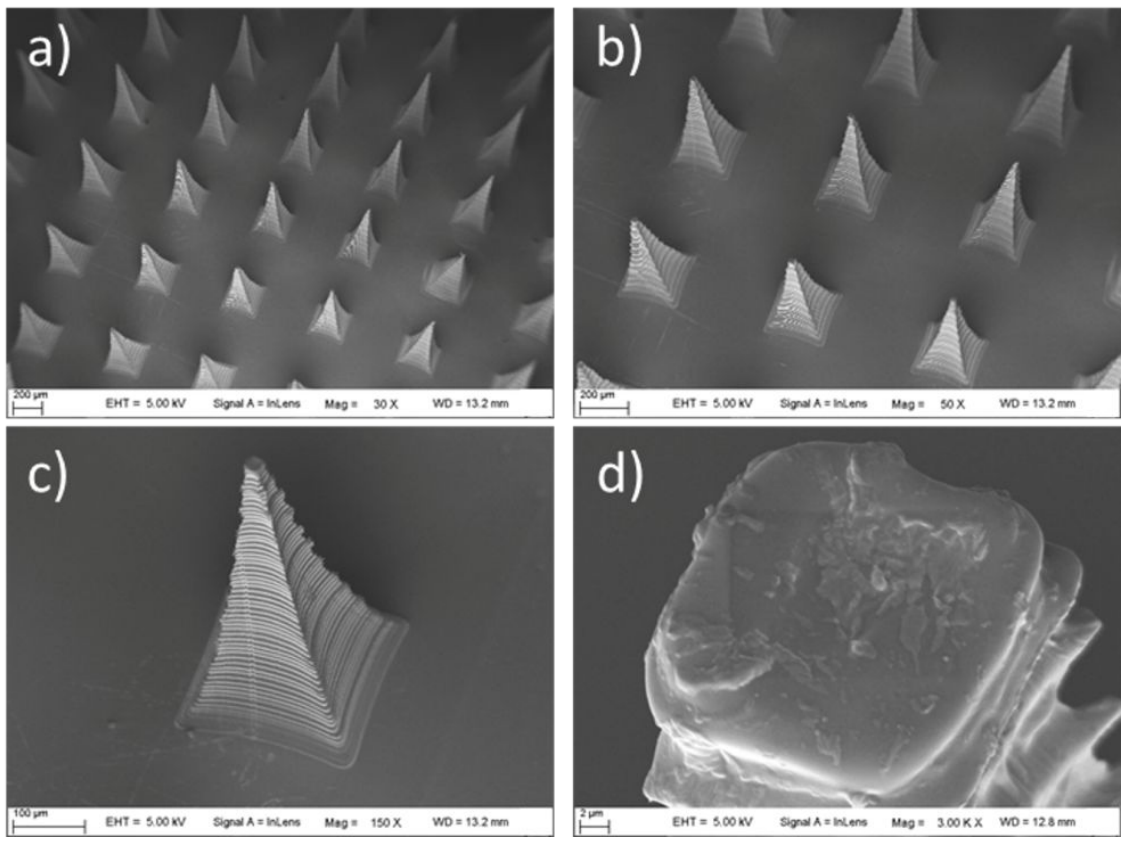

Figure S1. SEM images of CMC microneedle patches at a) 30, b) 50, c) 150, and d) 3000 times direct magnification. 

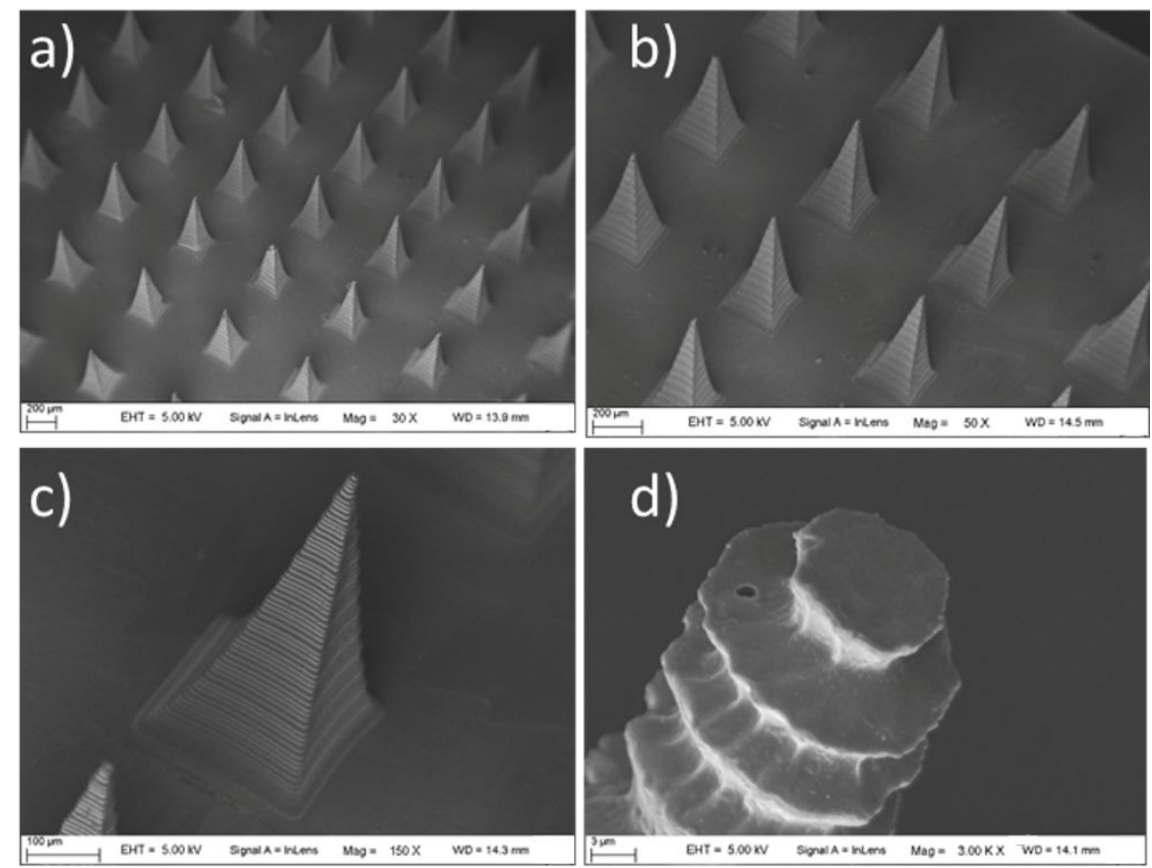

Figure S2. SEM images of HA microneedle patches at a) 30, b) 50, c) 150 , and d) 3000 times direct magnification.


Figure S3. Mapping graphene oxide (GO) in GO-PVP MN patches (500 mg/mL GO) with Raman spectroscopy. a) A microscope image of a randomly selected mapping area. The red sampling spots were randomly chosen for the Raman mapping. b) The $\mathrm{PVP} / \mathrm{GO}$ signal ratio distribution in $\mathrm{x}, \mathrm{y}$ coordinates. c) A representative Raman spectrum of the mapped area. 


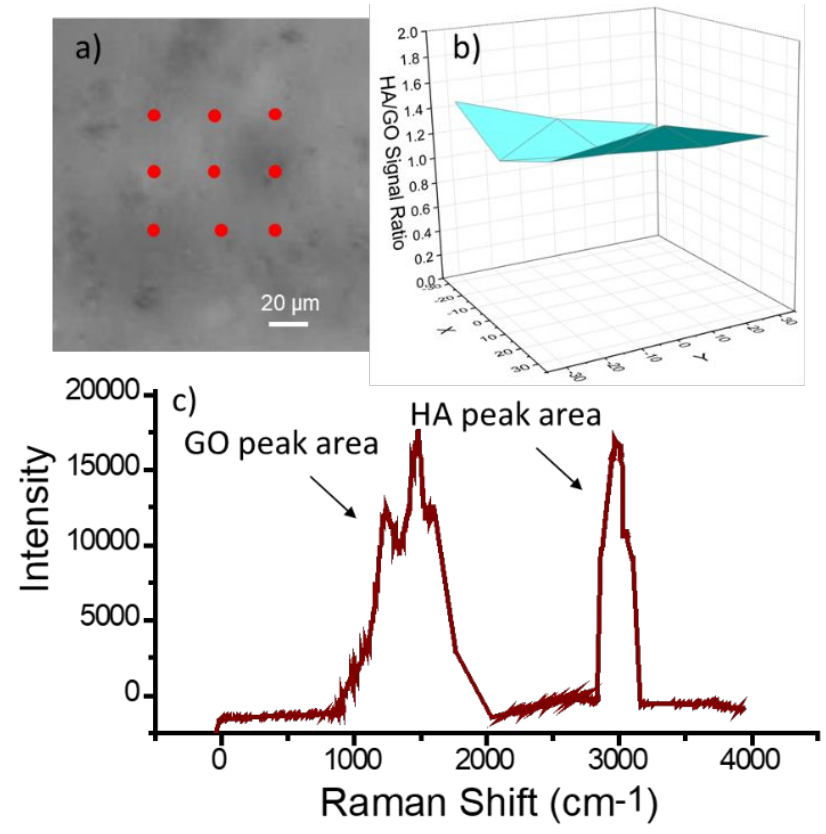

Figure S4. Mapping graphene oxide (GO) in GO-HA MN patches $(500 \mathrm{mg} / \mathrm{mL} \mathrm{GO})$ with Raman spectroscopy. a) A microscope image of a randomly selected mapping area. The red sampling spots were randomly chosen for the Raman mapping. b) The $\mathrm{HA} / \mathrm{GO}$ signal ratio distribution in $\mathrm{x}$, $\mathrm{y}$ coordinates. c) A representative Raman spectrum of the mapped area.

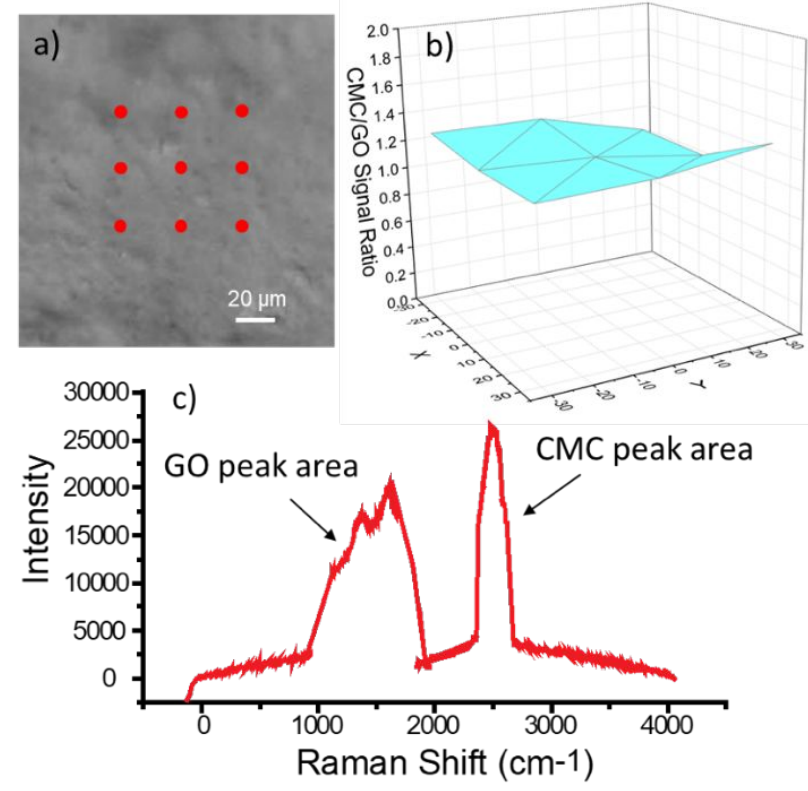

Figure S5. Mapping graphene oxide (GO) in GO-CMC MN patches (500 mg/mL GO) with Raman spectroscopy. a) A microscope image of a randomly selected mapping area. The red sampling spots were randomly chosen for the Raman mapping. b) The $\mathrm{CMC} / \mathrm{GO}$ signal ratio distribution in $\mathrm{x}$, y coordinates. c) A representative Raman spectrum of the mapped area. 

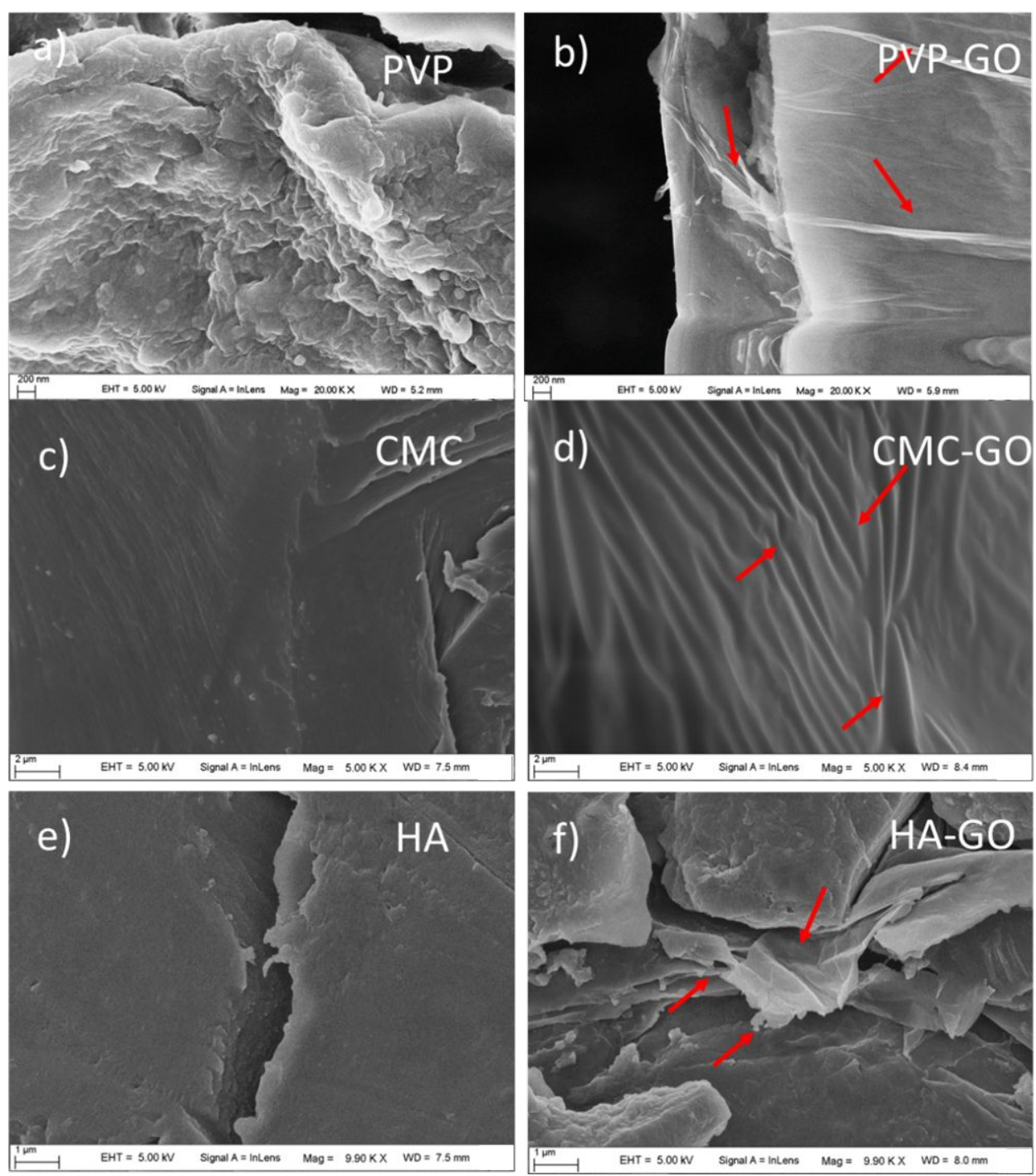

Figure S6. SEM cross sectional images of a) PVP, b) GO-PVP, c) CMC, d) GO-CMC, e) HA, and f) GO-HA microneedles. GO induced wrinkles are marked with red arrows. 
a)

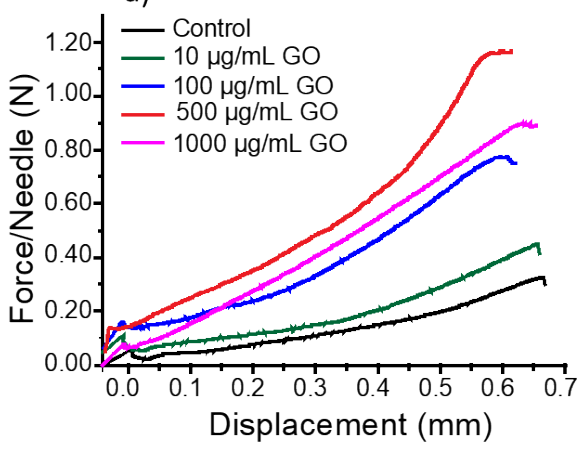

b)

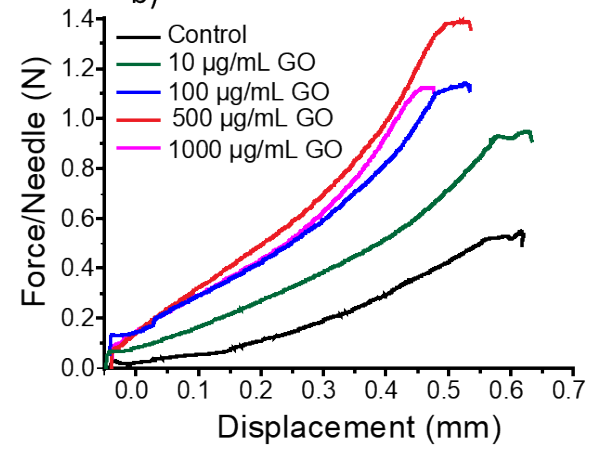

Figure S7. The mechanical strength of a) CMC and b) HA microneedles containing different GO concentrations.

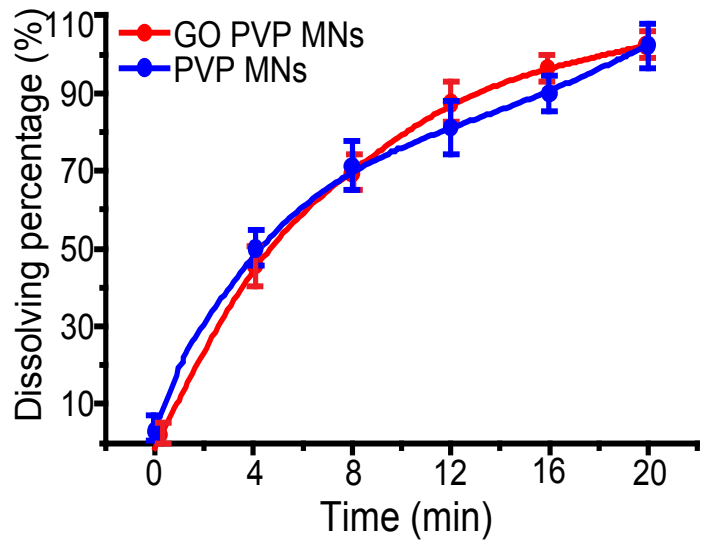

Figure S8. Dissolution kinetics of the PVP and GO-PVP microneedles.

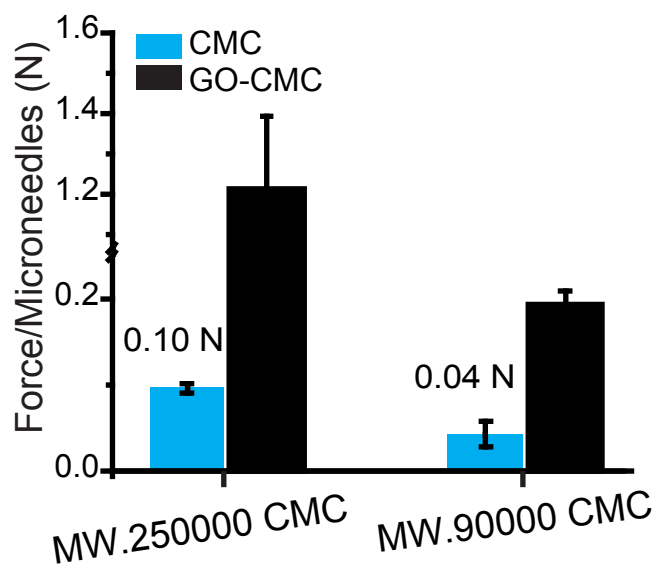

Figure S9. The mechanical strength of the microneedles made of CMC $250 \mathrm{~K}$ and CMC 90K with and without GO-doping (0.15 N required for skin penetration). 

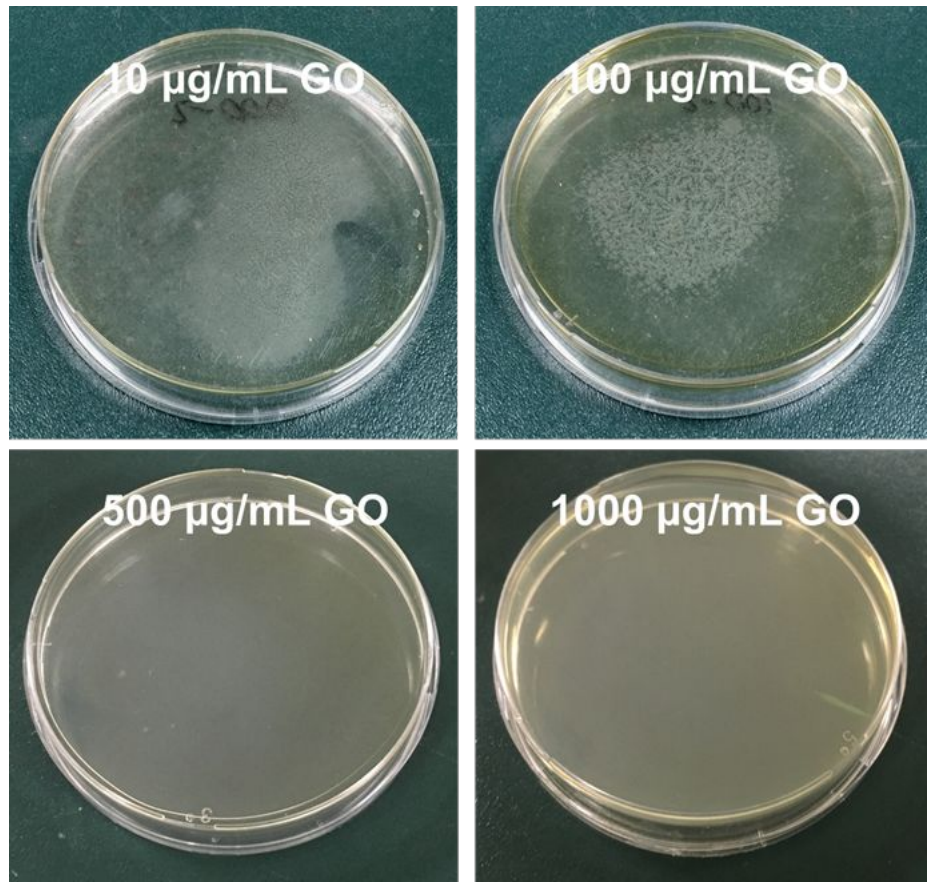

Figure S10. Agarose plates after mycete growth for $5 \mathrm{~d}$. Mycetes were collected by various GO-doped PVP microneedles that were exposed to ambient air for $3 \mathrm{~d}$.
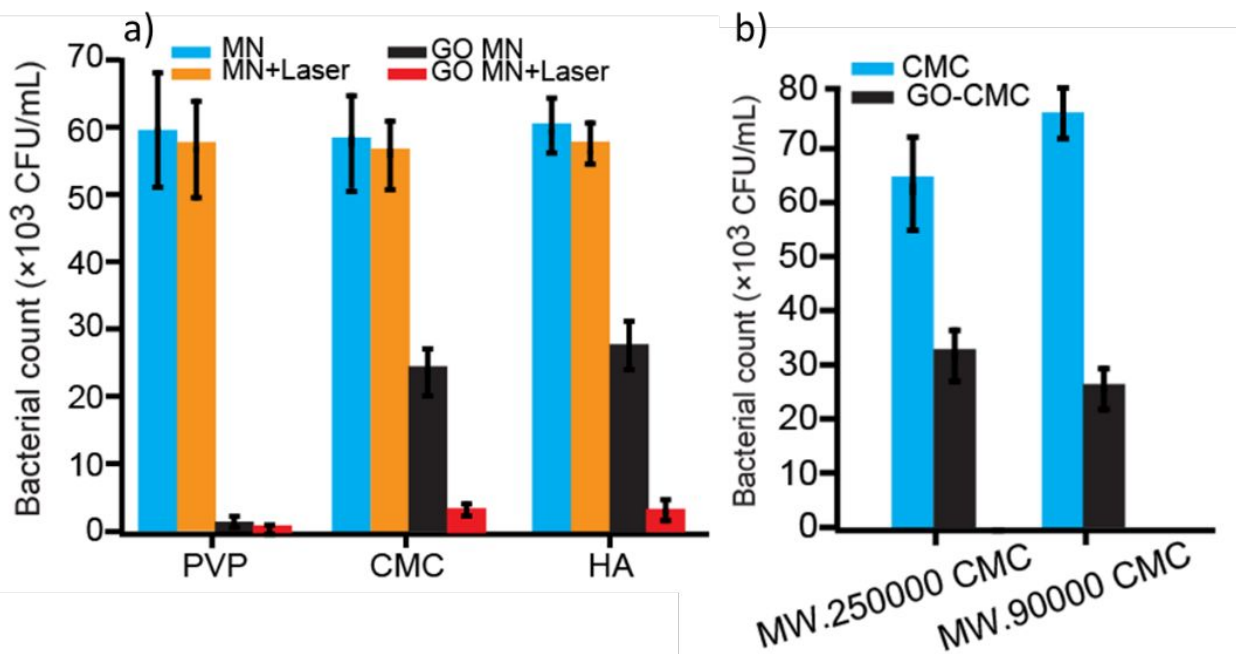

Figure S11. a) Quantitative analysis of E. coli colony-forming unit (CFUs) in contact with PVP, CMC, or HA MNs, with or without GO doping and with or without laser irradiation. b) Comparison of antibacterial effect of CMC MNs with different molecular weights with or without GO doping. 


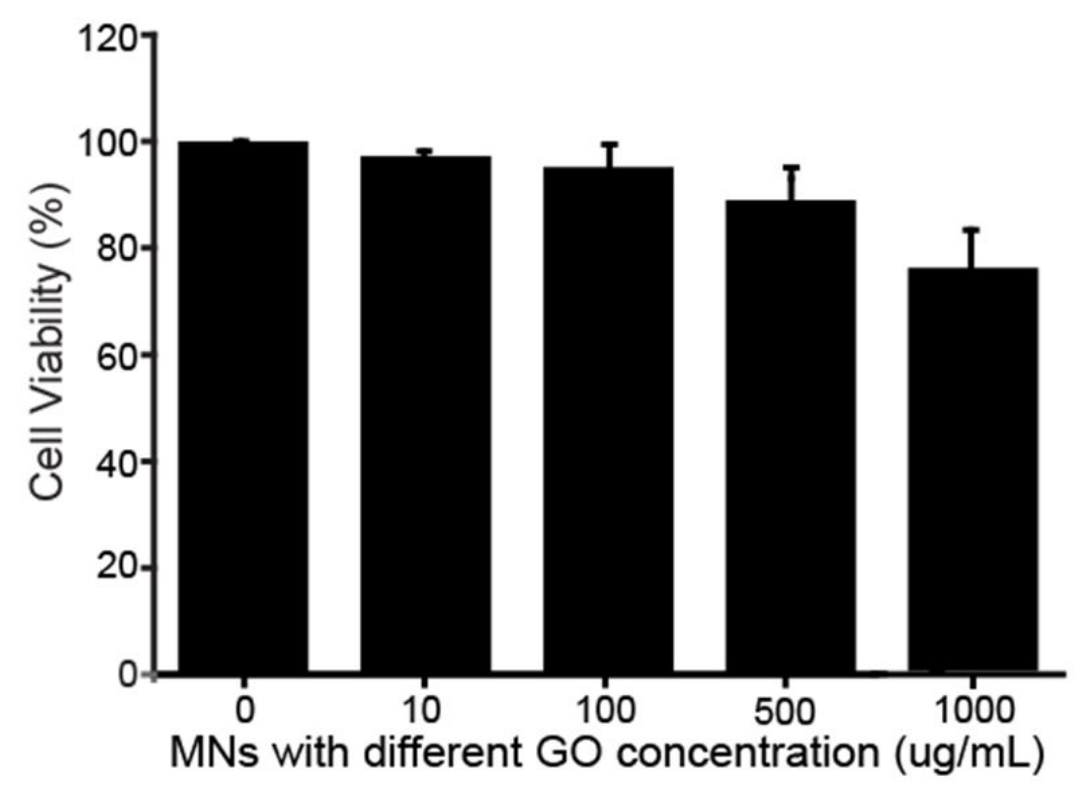

Figure S12. MTT assay of CCC-ESF cells treated with PVP MN solutions containing different GO concentrations.
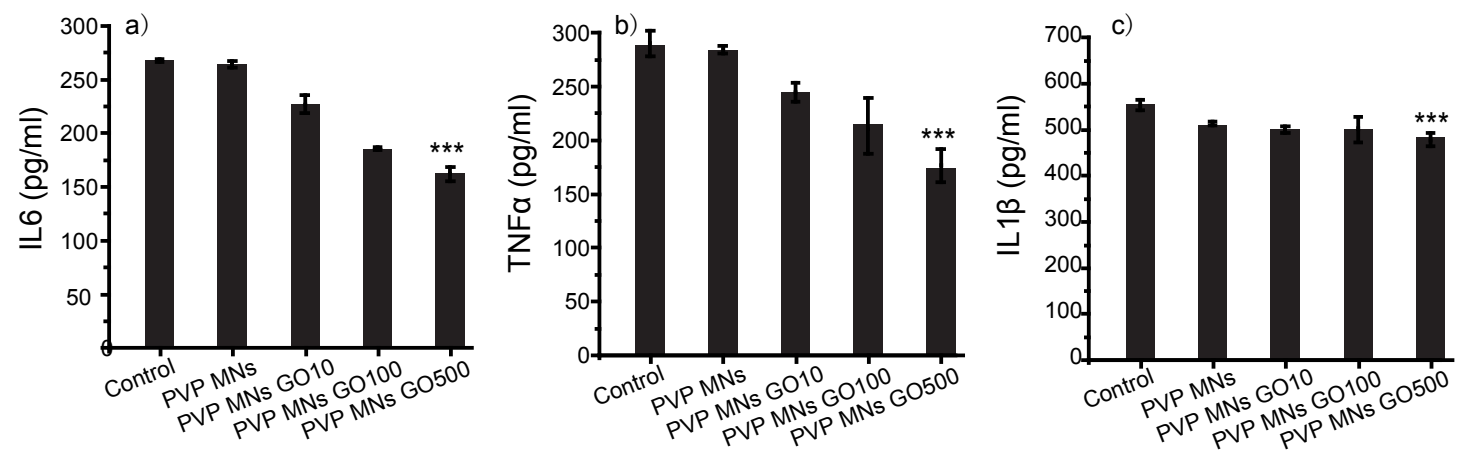

Figure S13. Activation response of M1 macrophages on GO-loaded PVP microneedles (MNs) and a control. The macrophages were incubated with PVP and GO-PVP MNs with different GO concentrations after LPS stimulation for the detection of a) IL6, b) TNF $\alpha$, and c) IL1 $\beta$ secretion in the culture medium. 



Figure S14. Liver toxicity determination. a) Plasma AST and b) ALT levels recorded from mice $24 \mathrm{~h}$ after different treatments.

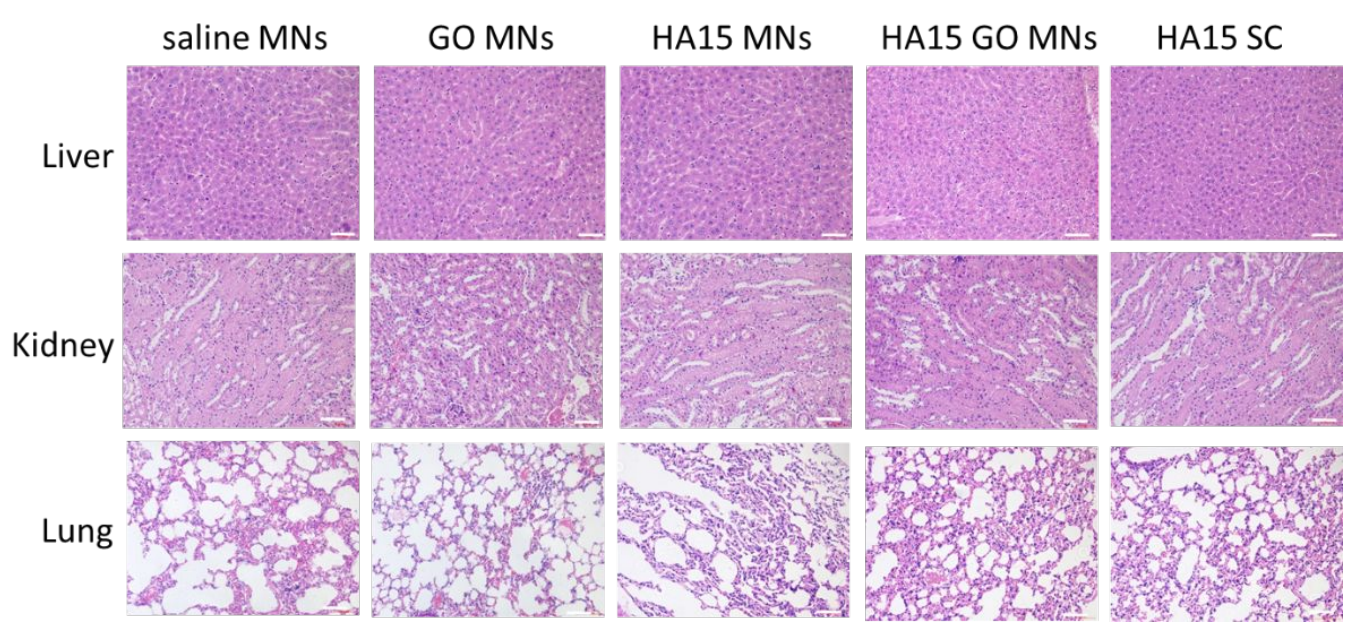

Figure S15. Histological study. H\&E staining of liver, kidney, and lung tissues treated with saline microneedles (MNs), GO, HA15, and HA15-GO MNs, and subcutaneous HA15. Scale bar: $200 \mu \mathrm{m}$. 